\title{
THE SUN AS A PULSATING ROTATING STAR*
}

\author{
KENNETH H. SCHATTEN
}

Victoria University of Wellington, Wellington, New Zealand

\begin{abstract}
Physical arguments are provided which suggest the following:
(a) The Sun rotates rapidly internally, with a period near one day. The arguments are based upon a low 'effective' plasma dynamic viscosity associated with a negative 'effective' magnetic density, $\varrho_{M}=-B^{2} / 4 \pi v^{2}$. This low (near zero) viscosity allows several calculations of the angular velocity of the solar core to be made. A fluid dynamical argument based upon the inviscous Navier-Stokes relation shows that for objects seated in a non-expanding magnetohydrodynamic fluid, the usual Kepler law should be replaced by $T^{2} \propto r^{4}$.

(b) The rapid rotation suggests that the solar magnetic field is deeply buried and, furthermore, that the Sun violates the Ferraro theorem. This violation results from a radial electric field necessary to support the solar plasma. This radial field requires the Sun to be charged with a charge $q=+2 \times 10^{11}$ esu. Thus the Sun's differential rotation may be viewed as arising from $\left.B^{\prime}\right]_{\theta}=0$ in the rest frame of the fluid where $\mathbf{E}_{r} \neq 0$ or alternatively as a polar spin down by the solar wind flow. Thus in the fluid frame, the solar activity cycle may be viewed as an Alfvén wave, with $T=\lambda / v_{\mathrm{A}}=20 \mathrm{yr}$. The velocity of material along the field is such that $\varrho v / B=$ const $\cong 4 \times 10^{-6} \mathrm{gm}$ $\left(\mathrm{G}^{-1} \mathrm{~cm}^{-2} \mathrm{~s}^{-1}\right)$, governed by the collapse of mass in the solar core; $(4 \mathrm{H}+4 \mathrm{e} \rightarrow 1 \mathrm{He}+$ $+2 \mathrm{e}$ ) results in a reduction of gas pressure unless $\sim 7 \times 10^{14} \mathrm{gm} \mathrm{s}^{-1}$ of material continually collapse so as to conserve the particle number in the solar interior.

(c) This requires the Sun to emit gravity waves with an energy comparable to its luminosity. The virial of the nuclear reactions in the core governs whether the energy goes into gravity waves or heat. A variable positron-electron core allows this.
\end{abstract}

* Due to lack of time, only the first part of this paper was presented. 